ASIAN JOURNAL OF MEDICAL SCIENCES

DOI:10.3126/ajms.v1i1.2927

\title{
Factors associated with nutritional status of the under five children Amita Pradhan ${ }^{a^{*}}$
}

\section{Department of Community Medicine, KIST Medical College, Lalitpur Nepal} \begin{abstract}
Objective: To identify factors associated to the nutritional status of under five children.

Method: The data used in this analysis are from Demographic and Health Survey 2006 conducted in Nepal. Total 5262 cases are included in the present study. A multinomial logistic regression model is used to study the relation between various factors and nutritional status.

Results: Increasing body mass index of mothers and wealth index shows decreasing likelihood of malnutrition among children. Rural children show insignificant higher likelihood of different forms of underweight and wasting as compared to urban area. Frequency of listening radio does not show significant association in case of mild and moderate wasting and shown very high unusual odds ratio in case of severe wasting. Similarly, lower frequency of watching television also does not show significantly higher likeliness of different form of stunting, underweight and wasting. Female children are more likely to be stunted, underweight and wasted as compared to male. Female headed households are more likely to have moderately and mildly stunted children and mixed results are observed for underweight and wasting. The likelihood for all forms of malnutrition is higher among children with smaller than average size at birth as compared to average or bigger size at birth. Mixed results are observed regarding likelihood of different forms of malnutrition among children with mothers having different educational level.
\end{abstract}

Conclusion: Body mass index of mothers is found significant variable while explaining children's nutritional status. Similarly, Size at birth is significantly associated with nutrition during the childhood.

Keywords: Stunting; wasting; under weight; under five children

\section{Introduction}

Nepalese children show evidences of under nutrition as indicated by their stunting, wasting or wasting and stunting combined along with the features of various micronutrient deficiency disorders. ${ }^{1}$ National surveys on nutritional status from 1975 to 1990 , showed the worse picture of nutrition in the country. National Family Health Survey (NFHS), 1996 in a nationally representative sample of children (6-36 months) showed that overall, 54.8\% were stunted, $12.7 \%$ showed wasting and $54.2 \%$ were underweight. Unpublished findings in 1998 indicate for the same age group 50.5\% were stunted, $8.5 \%$ showed wasting and $48.8 \%$ were underweight. Nepal Micronutrient Status Survey, 1998 showed that $54 \%$ of children in Nepal were stunted and $47 \%$ underweight. The first national nutritional survey in 1975 also showed similar findings of $48.1 \%$ stunted, $2.8 \%$ wasted and $50 \%$ underweight. The data suggest that there is no improvement in the nutritional status in the country during these two decades. ${ }^{2}$

If we look at data from Nepal Demographic and Health Survey (NDHS) 2001, the percent prevalence for underweight and wasted children of under five years of age are 48.3 and $9.6 \%$. Around $50 \%$ of the under five children are stunted. ${ }^{3}$ Similarly, NDHS 2006 reveals that the percent prevalence for underweight and wasted children of under five years of age are $39 \%$ and $13 \%$. Forty nine percent of the under five children are stunted. ${ }^{4}$ Looking at these figures we can say that rapid decline is not observed in stunting and slight decline in underweight can be seen. However percentage of wasted children shows increased trend.

Hence understanding the factors that affect the nutrition of children is a need. Various factors may play the role of predictor variables while explaining the state of malnutrition. This analysis tries to analyze the factors associated with nutritional status among children of under five years of age. This analysis focus on applying statistical methods to study the relation between outcome variables namely malnutrition in the form of stunting, wasting and underweight with predictor variables namely education and body mass index of the mothers, area and region of residence, sex of the child, sex of the household head, frequency of listening radio and watching television, size of the child at birth as described by the respondents.

\section{Materials and Methods}

The data used in this analysis are from the Demographic and Health Survey (DHS), 2006 conducted in Nepal. A total of 5262 cases are included in the present study.

\subsection{Statistics}

A multinomial logistic regression model is used to study the relation between various factors and nutritional status. Normal children are used as reference category. SPSS 13 is used to analyze the data.

\section{Results}

Table no. 1 shows that increasing body mass index (BMI) of mothers shows decreasing trend of odds ratios for mild (OR: .934; CI: .911, .958), moderate (OR: 924; CI: 886, 963) and severe (OR: 789; CI: 674, 923) stunting. Smaller size at birth shows increasing trend of odds ratios for mild (OR: 1.234; CI: $1.046,1.455)$, moderate (OR: 1.823; CI: $1.457,2.282$ ) and severe

\section{*Corresponding Autho}

Amita Pradhan, Associate professor, Department of Community Medi-

cine, KIST Medical College, Lalitpur Nepal, Ph. No. 9841302137

Email: amiseason@yahoo.com
(OR: 3.625 ; CI 1.876, 7.006) stunting as compared to average or bigger size at birth

Table no. 2 also shows similar pattern with higher BMI accompanying decreasing odds ratios for mild (OR :.890; CI: .863, .918), moderate ( OR: .757 ; CI: .728, .788) and severe (OR: .673; CI: .605, .748) underweight and smaller size at birth with increasing odds ratios for mild (OR: 1.444; CI: 1.131, 1.843), moderate (OR:3.120; CI: 2.417, 4.027) and severe ( OR: 4.363; CI: $2.740,6.946)$ underweight.

However, table no. 3 shows that BMI does not follow similar pattern of decreasing odds ratios in case of mild (OR: .844; CI: .822, .867), moderate (OR: .726; CI: .686, .769) and severe (OR: .859; CI: .708, 1.041) wasting. But smaller size at birth follows similar pattern with odds ratios for mild (OR 1.760; CI: 1.513, 2.047), moderate (OR: 2.318; CI: $1.787,3.006$ ) and severe (OR: 2.906; CI: 1.200, 7.037).

\section{Discussion}

Increasing body mass index (BMI) of mothers shows decreasing likelihood of all forms of stunting, underweight and wasting however in case of severe wasting the relationship is insignificant. Similarly, increasing wealth index of household shows decreasing likelihood except in case of severe stunting and wasting however the association is significant only in case of moderate stunting, moderate and severe underweight and moderate wasting. Mountain region children are found significantly higher for all forms of stunting whereas hill region children have significantly more likelihood in moderate and mild stunting as compared to terai. But no significant likelihood is observed in case of wasting and underweight. However moderately wasted children are significantly less likely in mountain and hill as compared to terai. Frequency of listening to radio has shown significant association in moderate stunting but in severe and mild stunting, it is not found significant. Similarly, less exposure to listening to radio has shown significantly higher odds for moderate and severe underweight. But frequency of listening radio does not show significant association in case of mild and moderate wasting and shown very high unusual odds ratio in case of severe wasting due to less number of cases who are severely wasted. Similarly, lower frequency of watching television also does not show significantly higher likeliness of different form of stunting, underweight and wasting.

Rural children are less likely to be severely, mildly and moderately stunted as compared to urban children, however the association is not significant. Rural children show insignificant higher likelihood of different forms of underweight and wasting as compared to urban area. In a study carried out by Saadah F. et. al. in 1999 in Indonesia, 32\% of rural children are malnourished against $27 \%$ of urban. ${ }^{5}$ This analysis shows similar results for underweight but mixed results for stunting and wasting.

Female children are more likely to be stunted as compared to male but the association is significant only in case of moderate stunting. Similarly, female are significantly more likely to be underweight and wasted as compared to male but association is significant only in case of moderate impairment.

Female headed households are more likely to have moderately and mildly stunted children but odds ratios are significant only in mild impairment However odds ratios show mixed and insignificant results in case of different forms of underweight and higher insignificant likelihood are observed for wasting. However, a study from Botswana conducted by Gobotswang K. 
A. Pradhan / Asian Journal of Medical Sciences, 1 (2010) 6-8

Table 1: Stunting among under five children according to various vari- Table 2: Underweight among under five children according to various ables

\begin{tabular}{|c|c|c|c|c|c|}
\hline \multirow[t]{2}{*}{ Stunting } & \multirow[t]{2}{*}{ Background variables } & \multirow[t]{2}{*}{ B } & \multirow[t]{2}{*}{$\begin{array}{l}\text { Odds } \\
\text { Ratio }\end{array}$} & \multicolumn{2}{|c|}{$\begin{array}{l}\text { 95\% CI for Odds } \\
\text { Ratio }\end{array}$} \\
\hline & & & & Lower & Upper \\
\hline \multirow{11}{*}{$\begin{array}{l}\text { Mildly } \\
\text { impaired }\end{array}$} & Intercept & .250 & & & \\
\hline & BMI of mother & -.068 & .934 & .911 & .958 \\
\hline & Wealth index factor score & -.084 & .919 & .823 & 1.028 \\
\hline & $\begin{array}{l}\text { Ecological region } \\
\text { Mountain } \\
\text { Hill } \\
\text { Terai * }\end{array}$ & $\begin{array}{l}.345 \\
.187\end{array}$ & $\begin{array}{l}1.412 \\
1.205\end{array}$ & $\begin{array}{l}1.155 \\
1.047\end{array}$ & $\begin{array}{l}1.727 \\
1.387\end{array}$ \\
\hline & $\begin{array}{l}\text { Do not listen radio } \\
\text { Listen less than once a week } \\
\text { Listen at least once a week } \\
\text { Listen almost every day* }\end{array}$ & $\begin{array}{l}-.042 \\
.058 \\
.026\end{array}$ & $\begin{array}{l}.959 \\
1.060 \\
1.026\end{array}$ & $\begin{array}{l}.745 \\
.903 \\
.865\end{array}$ & $\begin{array}{l}1.233 \\
1.244 \\
1.217\end{array}$ \\
\hline & $\begin{array}{l}\text { Do not watch television } \\
\text { Watch less than once a week } \\
\text { Watch at least once a week } \\
\text { Watch almost every day* }\end{array}$ & $\begin{array}{l}.118 \\
.061 \\
-.120\end{array}$ & $\begin{array}{l}1.125 \\
.063 \\
.887\end{array}$ & $\begin{array}{l}.895 \\
.862 \\
.689\end{array}$ & $\begin{array}{l}1.413 \\
1.310 \\
1.141\end{array}$ \\
\hline & $\begin{array}{l}\text { Area of residence } \\
\text { Rural } \\
\text { Urban * }\end{array}$ & .057 & 1.058 & .896 & 1.250 \\
\hline & $\begin{array}{l}\text { Sex of child } \\
\text { Female } \\
\text { Male* }\end{array}$ & .031 & 1.032 & .912 & 1.167 \\
\hline & $\begin{array}{l}\text { Size at birth } \\
\text { Smaller than average } \\
\text { Average and bigger* }\end{array}$ & .021 & 1.234 & 1.046 & 1.455 \\
\hline & $\begin{array}{l}\text { Sex of household head } \\
\text { Female } \\
\text { Male* }\end{array}$ & .177 & 1.193 & 1.023 & 1.393 \\
\hline & $\begin{array}{l}\text { Education of mother } \\
\text { No education } \\
\text { Primary } \\
\text { Secondary } \\
\text { Higher* } \\
\end{array}$ & $\begin{array}{l}1.479 \\
1.346 \\
.806\end{array}$ & $\begin{array}{l}4.387 \\
3.841 \\
2.239\end{array}$ & $\begin{array}{l}2.808 \\
2.450 \\
1.455\end{array}$ & $\begin{array}{l}6.852 \\
6.021 \\
3.445\end{array}$ \\
\hline \multirow{11}{*}{$\begin{array}{l}\text { Moder- } \\
\text { ately } \\
\text { impaired }\end{array}$} & Intercept & -2.070 & & & \\
\hline & BMI of mother & -.079 & .924 & .886 & .963 \\
\hline & Wealth index factor score & -.316 & .729 & .581 & .915 \\
\hline & $\begin{array}{l}\text { Ecological region } \\
\text { Mountain } \\
\text { Hill } \\
\text { Terai * }\end{array}$ & $\begin{array}{l}.525 \\
.420\end{array}$ & $\begin{array}{l}1.690 \\
1.522\end{array}$ & $\begin{array}{l}1.263 \\
1.219\end{array}$ & $\begin{array}{l}2.263 \\
1.900\end{array}$ \\
\hline & $\begin{array}{l}\text { Do not listen radio } \\
\text { Listen less than once a week } \\
\text { Listen at least once a week } \\
\text { Listen almost every day* }\end{array}$ & $\begin{array}{l}.687 \\
.490 \\
.455\end{array}$ & $\begin{array}{l}1.987 \\
1.633 \\
1.576\end{array}$ & $\begin{array}{l}1.369 \\
1.248 \\
1.179\end{array}$ & $\begin{array}{l}2.883 \\
2.137 \\
2.107\end{array}$ \\
\hline & $\begin{array}{l}\text { Do not watch television } \\
\text { Watch less than once a week } \\
\text { Watch at least once a week } \\
\text { Watch almost every day* }\end{array}$ & $\begin{array}{l}.376 \\
.362 \\
-.070\end{array}$ & $\begin{array}{l}1.456 \\
1.437 \\
.932\end{array}$ & $\begin{array}{l}.963 \\
.970 \\
.567\end{array}$ & $\begin{array}{l}2.200 \\
2.128 \\
1.533\end{array}$ \\
\hline & $\begin{array}{l}\text { Area of residence } \\
\text { Rural } \\
\text { Urban * }\end{array}$ & -.094 & .910 & .698 & 1.188 \\
\hline & $\begin{array}{l}\text { Sex of child } \\
\text { Female } \\
\text { Male * }\end{array}$ & .191 & 1.211 & 1.003 & 1.462 \\
\hline & $\begin{array}{l}\text { Size at birth } \\
\text { Smaller than average } \\
\text { Average and bigger* }\end{array}$ & .601 & 1.823 & 1.457 & 2.282 \\
\hline & $\begin{array}{l}\text { Sex of household head } \\
\text { Female } \\
\text { Male* }\end{array}$ & .183 & 1.200 & .954 & 1.511 \\
\hline & $\begin{array}{l}\text { Education of mother } \\
\text { No education } \\
\text { Primary } \\
\text { Secondary } \\
\text { Higher* }\end{array}$ & $\begin{array}{l}1.799 \\
1.389 \\
.401\end{array}$ & $\begin{array}{l}6.041 \\
4.012 \\
1.493\end{array}$ & $\begin{array}{l}1.817 \\
1.200 \\
.444\end{array}$ & $\begin{array}{l}20.086 \\
13.413 \\
5.019\end{array}$ \\
\hline \multirow{11}{*}{$\begin{array}{l}\text { Severely } \\
\text { impaired }\end{array}$} & Intercept & .018 & & & \\
\hline & BMI of mother & -.237 & .789 & .674 & .923 \\
\hline & Wealth index factor score & .063 & 1.065 & .508 & 2.234 \\
\hline & $\begin{array}{l}\text { Ecological region } \\
\text { Mountain } \\
\text { Hill } \\
\text { Terai * } \\
\end{array}$ & $\begin{array}{l}.966 \\
.073\end{array}$ & $\begin{array}{l}2.629 \\
.929\end{array}$ & $\begin{array}{l}1.127 \\
.405\end{array}$ & $\begin{array}{l}6.132 \\
2.134\end{array}$ \\
\hline & $\begin{array}{l}\text { Do not listen radio } \\
\text { Listen less than once a week } \\
\text { Listen at least once a week } \\
\text { Listen almost every day* }\end{array}$ & $\begin{array}{l}.666 \\
.210 \\
.451\end{array}$ & $\begin{array}{l}1.946 \\
.811 \\
1.570\end{array}$ & $\begin{array}{l}.660 \\
.308 \\
.594\end{array}$ & $\begin{array}{l}5.738 \\
2.135 \\
4.149\end{array}$ \\
\hline & $\begin{array}{l}\text { Do not watch television } \\
\text { Watch less than once a week } \\
\text { Watch at least once a week } \\
\text { Watch almost every day* }\end{array}$ & $\begin{array}{l}1.112 \\
.351 \\
-.703\end{array}$ & $\begin{array}{l}3.041 \\
1.420 \\
.495\end{array}$ & $\begin{array}{l}.687 \\
.327 \\
.050\end{array}$ & $\begin{array}{l}13.463 \\
6.177 \\
4.914\end{array}$ \\
\hline & $\begin{array}{l}\text { Area of residence } \\
\text { Rural } \\
\text { Urban * }\end{array}$ & -.330 & .719 & .297 & 1.737 \\
\hline & $\begin{array}{l}\text { Sex of child } \\
\text { Female } \\
\text { Male* }\end{array}$ & .337 & 1.401 & .736 & 2.667 \\
\hline & $\begin{array}{l}\text { Size at birth } \\
\text { Smaller than average } \\
\text { Average and bigger* }\end{array}$ & 1.288 & 3.625 & 1.876 & 7.006 \\
\hline & $\begin{array}{l}\text { Sex of household head } \\
\text { Female } \\
\text { Male* }\end{array}$ & -.936 & .392 & .119 & 1.292 \\
\hline & $\begin{array}{l}\text { Education of mother } \\
\text { No education } \\
\text { Primary } \\
\text { Secondary } \\
\text { Higher* }\end{array}$ & $\begin{array}{l}.152 \\
-1.153 \\
-.507\end{array}$ & $\begin{array}{l}1.164 \\
.316 \\
.602\end{array}$ & $\begin{array}{l}.113 \\
.023 \\
.062\end{array}$ & $\begin{array}{l}12.023 \\
4.334 \\
5.811\end{array}$ \\
\hline
\end{tabular}
variables

\begin{tabular}{|c|c|c|c|c|c|}
\hline \multirow[t]{2}{*}{$\begin{array}{l}\text { Under- } \\
\text { weight }\end{array}$} & \multirow[t]{2}{*}{ Background variables } & \multirow[t]{2}{*}{ B } & \multirow[t]{2}{*}{$\begin{array}{l}\text { Odds } \\
\text { Ratio }\end{array}$} & \multicolumn{2}{|c|}{$\begin{array}{lcc}95 \% & \text { CI } & \text { for } \\
\text { Odds } & \text { Ratio } & \\
\end{array}$} \\
\hline & & & & Lower & Upper \\
\hline \multirow{12}{*}{$\begin{array}{l}\text { 1st degree } \\
\text { Mild malnu- } \\
\text { trition }\end{array}$} & Intercept & 2.855 & & & \\
\hline & BMI of mother & -.116 & .890 & .863 & .918 \\
\hline & Wealth index factor score & -.105 & .901 & .790 & 1.027 \\
\hline & $\begin{array}{l}\text { Ecological region } \\
\text { Mountain } \\
\text { Hill } \\
\text { Terai * } \\
\end{array}$ & $\begin{array}{l}.065 \\
.074\end{array}$ & $\begin{array}{l}1.067 \\
1.076\end{array}$ & $\begin{array}{l}.826 \\
.899\end{array}$ & $\begin{array}{l}1.378 \\
1.289\end{array}$ \\
\hline & Do not listen radio & .214 & 1.239 & .873 & 1.759 \\
\hline & $\begin{array}{l}\text { Listen less than once a week } \\
\text { Listen at least once a week } \\
\text { Listen almost every day* }\end{array}$ & $\begin{array}{l}.103 \\
.215\end{array}$ & $\begin{array}{l}1.109 \\
1.240\end{array}$ & $\begin{array}{l}.905 \\
.999\end{array}$ & $\begin{array}{l}1.359 \\
1.540\end{array}$ \\
\hline & $\begin{array}{l}\text { Do not watch television } \\
\text { Watch less than once a week } \\
\text { Watch at least once a week } \\
\text { Watch almost every day* }\end{array}$ & $\begin{array}{l}.151 \\
.068 \\
-.190\end{array}$ & $\begin{array}{l}1.163 \\
1.070 \\
.827\end{array}$ & $\begin{array}{l}.867 \\
.824 \\
.610\end{array}$ & $\begin{array}{l}1.560 \\
1.391 \\
1.120\end{array}$ \\
\hline & $\begin{array}{l}\text { Area of residence } \\
\text { Rural } \\
\text { Urban * }\end{array}$ & .061 & 1.063 & .862 & 1.311 \\
\hline & $\begin{array}{l}\text { Sex of child } \\
\text { Female } \\
\text { Male* }\end{array}$ & .004 & 1.004 & .855 & 1.178 \\
\hline & $\begin{array}{l}\text { Size at birth } \\
\text { Smaller than average } \\
\text { Average and bigger* }\end{array}$ & .367 & 1.444 & 1.131 & 1.843 \\
\hline & $\begin{array}{l}\text { Sex of household head } \\
\text { Female } \\
\text { Male* }\end{array}$ & .079 & 1.082 & .888 & 1.318 \\
\hline & $\begin{array}{l}\text { Education of mother } \\
\text { No education } \\
\text { Primary } \\
\text { Secondary } \\
\text { Higher* } \\
\end{array}$ & $\begin{array}{l}.515 \\
.362 \\
.111\end{array}$ & $\begin{array}{l}1.673 \\
1.436 \\
1.117\end{array}$ & $\begin{array}{l}1.060 \\
.905 \\
.728\end{array}$ & $\begin{array}{l}2.642 \\
2.279 \\
1.713\end{array}$ \\
\hline \multirow{15}{*}{$\begin{array}{l}\text { 2nd degree } \\
\text { Moderate } \\
\text { malnutrition }\end{array}$} & Intercept & 4.755 & & & \\
\hline & BMI of mother & -.278 & .757 & .728 & .788 \\
\hline & Wealth index factor score & -.313 & .732 & .612 & .875 \\
\hline & $\begin{array}{l}\text { Ecological region } \\
\text { Mountain } \\
\text { Hill } \\
\text { Terai * }\end{array}$ & $\begin{array}{l}-.125 \\
.029\end{array}$ & $\begin{array}{l}.883 \\
1.029\end{array}$ & $\begin{array}{l}.659 \\
.834\end{array}$ & $\begin{array}{l}1.182 \\
1.270\end{array}$ \\
\hline & Do not listen radio & .556 & 1.743 & 1.187 & 2.560 \\
\hline & & $\begin{array}{l}.278 \\
.242\end{array}$ & $\begin{array}{l}1.321 \\
1.274\end{array}$ & $\begin{array}{l}1.041 \\
.984\end{array}$ & $\begin{array}{l}1.677 \\
1.651\end{array}$ \\
\hline & Listen almost every day* & & & & \\
\hline & Do not watch television & .305 & 1.357 & .956 & 1.925 \\
\hline & Watch less than once a week & .173 & 1.189 & .863 & 1.639 \\
\hline & $\begin{array}{l}\text { Watch at least once a week } \\
\text { Watch almost every day* }\end{array}$ & -.198 & .820 & .557 & 1.208 \\
\hline & $\begin{array}{l}\text { Area of residence } \\
\text { Rural } \\
\text { Urban * }\end{array}$ & .174 & 1.190 & .927 & 1.528 \\
\hline & $\begin{array}{l}\text { Sex of child } \\
\text { Female } \\
\text { Male* }\end{array}$ & .229 & 1.257 & 1.046 & 1.511 \\
\hline & $\begin{array}{l}\text { Size at birth } \\
\text { Smaller than average } \\
\text { Average and bigger* }\end{array}$ & 1.138 & 3.120 & 2.417 & 4.027 \\
\hline & $\begin{array}{l}\text { Sex of household head } \\
\text { Female } \\
\text { Male* }\end{array}$ & .142 & 1.153 & .920 & 1.445 \\
\hline & $\begin{array}{l}\text { Education of mother } \\
\text { No education } \\
\text { Primary } \\
\text { Secondary } \\
\text { Higher* }\end{array}$ & $\begin{array}{l}.698 \\
.255 \\
-.040\end{array}$ & $\begin{array}{l}2.009 \\
1.290 \\
.961\end{array}$ & $\begin{array}{l}1.003 \\
.639 \\
.489\end{array}$ & $\begin{array}{l}4.025 \\
2.604 \\
1.891\end{array}$ \\
\hline \multirow{15}{*}{$\begin{array}{l}\text { 3rd degree } \\
\text { Severe mal- } \\
\text { nutrition }\end{array}$} & Intercept & 4.772 & & & \\
\hline & BMI of mother & -.397 & .673 & .605 & .748 \\
\hline & Wealth index factor score & -.623 & .536 & .298 & .964 \\
\hline & $\begin{array}{l}\text { Ecological region } \\
\text { Mountain } \\
\text { Hill } \\
\text { Terai * }\end{array}$ & $\begin{array}{l}-.336 \\
-.568\end{array}$ & $\begin{array}{l}.714 \\
.567\end{array}$ & $\begin{array}{l}.374 \\
.333\end{array}$ & $\begin{array}{l}1.363 \\
.963\end{array}$ \\
\hline & Do not listen radio & 1.075 & 2.929 & 1.224 & 7.007 \\
\hline & Listen less than once a week & .854 & 2.350 & 1.177 & 4.691 \\
\hline & $\begin{array}{l}\text { Listen at least once a week } \\
\text { Listen almost every day* }\end{array}$ & 1.117 & 3.055 & 1.493 & 6.250 \\
\hline & Do not watch television & -.017 & .983 & .427 & 2.262 \\
\hline & Watch less than once a week & -.261 & .770 & .345 & 1.719 \\
\hline & $\begin{array}{l}\text { Watch at least once a week } \\
\text { Watch almost every day* }\end{array}$ & -.871 & .418 & .137 & 1.281 \\
\hline & $\begin{array}{l}\text { Area of residence } \\
\text { Rural } \\
\text { Urban * }\end{array}$ & .315 & 1.370 & .731 & 2.566 \\
\hline & $\begin{array}{l}\text { Sex of child } \\
\text { Female } \\
\text { Male * }\end{array}$ & .227 & 1.255 & .829 & 1.900 \\
\hline & $\begin{array}{l}\text { Size at birth } \\
\text { Smaller than average } \\
\text { Average and bigger* }\end{array}$ & 1.473 & 4.363 & 2.740 & 6.946 \\
\hline & $\begin{array}{l}\text { Sex of household head } \\
\text { Female } \\
\text { Male* }\end{array}$ & -.180 & .835 & .477 & 1.461 \\
\hline & $\begin{array}{l}\text { Education of mother } \\
\text { No education } \\
\text { Primary } \\
\text { Secondary } \\
\text { Higher* } \\
\end{array}$ & $\begin{array}{l}.146 \\
-.684 \\
-.790\end{array}$ & $\begin{array}{l}1.157 \\
.505 \\
.454\end{array}$ & $\begin{array}{l}.133 \\
.055 \\
.051\end{array}$ & $\begin{array}{l}10.045 \\
4.601 \\
4.017\end{array}$ \\
\hline
\end{tabular}

found that female headed households are 1.5 times $(\mathrm{p}=016)$ more likely to be ( ferent forms of stunting, underweight and wasting. The likelihood for all forms of malnutrition is higher among children with smaller than average size at birth as compared to average or bigger size at birth. The association of size at birth with nutritional status is also supported by the study carried out in Malawi by Madise N. J. and Mpoma Mabel.

Level of education of mothers is found significantly associated with mild stunting however in case of moderate stunting mixed results are observed and in case of severe stunting association is insignificant.

Mixed results are observed regarding likelihood of different forms of underweight among children with mothers having different level of education. 
Table 3: Wasting among under five children according to various vari- Malawi by Madise N. J. and Mpoma Mabel5,7 the educational effect is found

ables

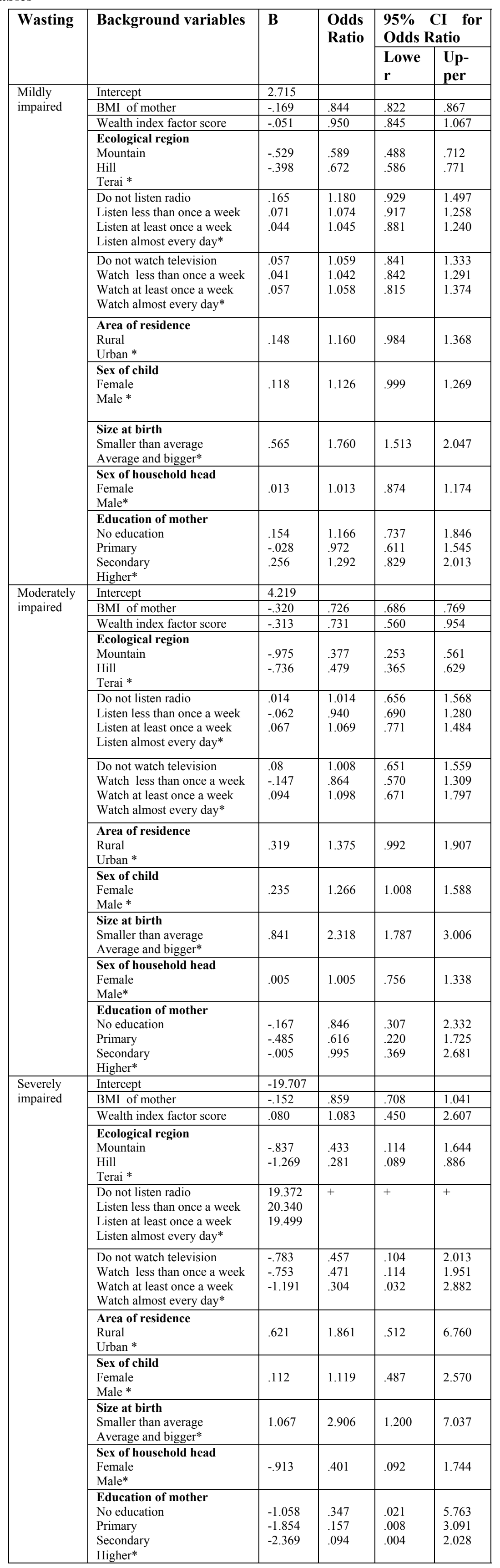

* Reference category; + very high unusual odds observed

But in case of moderate and severe wasting lower educational level of mothers is found associated with less likelihood however statistical significance is not seen. In studies carried out in Indonesia by Saadah F. et. Al. in 1999 and in

\section{to be statistically significant.}

\section{Conclusion}

Wealth index of household shows mixed results while examining the relation with different forms of malnutrition. So along with the economic status of household, the food pattern may need to be examined because irrespective of the economic condition, the food of choice for the children may have different nutritional value. Body mass index of mothers is found significant variable while explaining children's nutritional status. Similarly, Size at birth is significantly associated with nutrition during the childhood. So more emphasis shall be given to improve the nutritional status of women during pregnancy and lactation.

\section{References}

1. Adhikari RK, Krantz ME. Child Nutrition and Health. III ed., HLMC, IOM, Nepal, 2001,pp 21-22.

2. World Health Organization. Nutrition in South East Asia. WHO Regional Office for South East Asia. New Delhi, 2000.

3. Ministry of Health and Population, New Era, ORC Macro. Nepal Demographic and Health Survey. 2001.

4. Ministry of Health and Population, New Era, ORC Macro. Nepal Demographic and Health Survey. 2006.

5. Saadah F,Waters H and Heywood P. Indonesia: Under nutrition in Young Children. East Asia and the Pacific Region Watching Brief 1999; (1).

6. Gobotswang K. Determinants of the Nutritional Status of Children in a Rural African Setting: The Case of Chobe District, Botswana. (Cited in June 2009). Available from: URL: www.Unu.edu/unupress/food/v191e/ cho9.htm

7. Madise NJ and Mpoma M. Child Malnutrition and Feeding Practices in Malawi. (Cited in June 2009). Available from: URL: www.Unu.edu/ unupress/food/v182e/begin.htm 